\title{
Salinomycin exhibits anti-angiogenic activity against human glioma in vitro and in vivo by suppressing the VEGF-VEGFR2-AKT/FAK signaling axis
}

\author{
YAN-LING BI ${ }^{1 *}$, PEI-YAN MI ${ }^{1 *}$, SHI-JUN ZHAO ${ }^{2}$, HENG-MING PAN ${ }^{3}$, HUI-JUAN LI ${ }^{1}$, FEI LIU ${ }^{4}$, \\ LU-RONG SHAO ${ }^{2}$, HUI-FANG ZHANG ${ }^{2}$, PU ZHANG $^{1}$ and SHI-LIANG JIANG ${ }^{3}$ \\ ${ }^{1}$ Department of Cardiology, Taian Central Hospital; ${ }^{2}$ Key Laboratory of Cerebral Microcirculation \\ in Universities of Shandong, Taishan Medical University, Taian, Shandong 271000; ${ }^{3}$ Department of Cardiology, \\ Shandong Provincial Hospital Affiliated to Shandong University, Jinan, Shandong 250021; \\ ${ }^{4}$ Department of Breast Surgery, Taian Central Hospital, Taian, Shandong 271000, P.R. China
}

Received November 2, 2016; Accepted March 27, 2017

DOI: $10.3892 / \mathrm{ijmm} .2017 .2940$

\begin{abstract}
Tumor angiogenesis plays a crucial role in tumor growth, progression and metastasis, and suppression of tumor angiogenesis has been considered as a promising anticancer strategy. Salinomycin (SAL), an antibiotic, displays novel anticancer potential against several human cancer cells in vitro and in vivo. However, little information concerning its anti-angiogenic properties is available. Therefore, the anti-angiogenic effect of SAL and the underlying mechanism in human glioma were evaluated in the present study. The results indicated that SAL treatment significantly inhibited human umbilical vein endothelial cell (HUVEC) proliferation, migration, invasion and capillary-like tube formation. Further investigation on intracellular mechanisms showed that SAL markedly suppressed FAK and AKT phosphorylation, and downregulated vascular endothelial growth factor (VEGF) expression in HUVECs. Pretreatment of cells with a PI3K inhibitor (LY294002) and FAK inhibitor (PF562271) markedly enhanced SAL-induced inhibition of HUVEC proliferation and migration, respectively. Moreover, U251 human glioma xenograft growth was also effectively blocked by SAL treatment in vivo via inhibition of angiogenesis involving FAK and AKT dephosphorylation. Taken together, our findings validated that SAL inhibits angiogenesis and human glioma growth through suppression of the VEGF-VEGFR2-AKT/FAK signaling axis, indicating the potential application of SAL for the treatment of human glioma.
\end{abstract}

Correspondence to: Dr Shi-Liang Jiang, Department of Cardiology, Shandong Provincial Hospital Affiliated to Shandong University, 324 Jingwu Weiqi Road, Jinan, Shandong 250021, P.R. China

E-mail: slyyjsl@126.com

*Contributed equally

Key words: salinomycin, migration, invasion, angiogenesis, glioma

\section{Introduction}

Tumor progression relies on a series of sequential events, including tumor initiation, growth, angiogenesis and metastasis, which occur in a complex and dynamic microenvironment and are regulated by a number of mediators and signaling transduction pathways (1-3). Growing evidence indicates the crucial role of angiogenesis in tumor growth and metastasis $(4,5)$. Tumor cells can develop their microenvironment by the secretion of vascular endothelial growth factor (VEGF) or cytokines to promote abnormal tumor neovasculature formation, which can provide nutrients for tumor growth and a route for metastasis (6). Owing to the key role of angiogenesis, inhibition of tumor angiogenesis has been considered as an effective strategy for suppressing tumor progression $(7,8)$. It has also been demonstrated that agents with anti-angiogenic properties can effectively inhibit tumor cell growth and metastasis (9). Thus, searching for novel agents with anti-angiogenic activity represents an effective strategy for the overall control of human cancers.

Salinomycin (SAL), a polyether antibiotic, isolated from bacterium Streptomyces albus, has been used extensively to improve the nutrient absorption and feeding efficiency of poultry (10). SAL was identified as a potent anticancer agent among 16,000 compounds against human breast cancer stem cells (CSCs) for the first time in 2009 (11). It was also demonstrated that SAL displays antitumor activities in other types of human CSCs, including colorectal, lung, gastric, pancreatic and osteosarcoma CSCs (12-16). Recently, several studies have shown that SAL displays broad-spectrum anticancer properties, including inhibition of proliferation, migration and invasion, and induction of autophagy and apoptosis in cancer cells (17-21). Additionally, accumulating evidence indicates that SAL can enhance the cytotoxic effects of several chemotherapeutic drugs, such as doxorubicin and etoposide $(22,23)$. An increasing number of studies have reported that induction of DNA damage (23), mitochondrial dysfunction (24), reactive oxygen species (ROS) accumulation (20), signal regulation of FOXO3a, Wnt/ $\beta$-catenin, 
STAT3/Skp2 and Akt/NF-kB/mTOR (25-28), all contribute to SAL-mediated anticancer mechanisms. However, little information concerning the anti-angiogenic potential of SAL is available. In this study, SAL-mediated angiogenic activity was examined, and the underlying molecular mechanisms in human glioma were also evaluated.

\section{Materials and methods}

Materials. SAL and conventional chemicals were all obtained from Sigma-Aldrich (St. Louis, MO, USA). Dulbecco's modified Eagle's medium (DMEM)-F12 and fetal bovine serum (FBS) were purchased from Beyotime (Beijing, China). All of the antibodies and inhibitors (LY294002 and PF-562271) used in this study were purchased from Cell Signaling Technology, Inc. (Beverly, MA, USA). All solvents used were of high-performance liquid chromatography (HPLC) grade. Water used in this study was obtained from a Milli-Q from Millipore (Billerica, MA, USA).

Cell culture. The U251 human glioma cell line and human umbilical vein endothelial cells (HUVECs) were obtained from the American Type Culture Collection (ATCC; Manassas, VA, USA). HUVECs were cultured in DMEM-F12 supplemented with FBS (10\%) at $37^{\circ} \mathrm{C}$ in a humidified incubator with $5 \% \mathrm{CO}_{2}$ atmosphere.

Measurement of cell viability (MTT assay). The effect of SAL on HUVEC growth was determined by MTT assay. Briefly, HUVECs $(6,000$ cells/well) were seeded in 96-well culture plates for $24 \mathrm{~h}$ and incubated with different concentrations of SAL. In the preliminary experiments, SAL treatment for 12 , 24,48 and $72 \mathrm{~h}$ showed time-dependent effects on cell growth inhibition. However, treatment for $48 \mathrm{~h}$ was the optimal time and was selected for further mechanism evaluation. After SAL treatment for $48 \mathrm{~h}, 20 \mu \mathrm{l} /$ well of MTT solution $(5 \mathrm{mg} / \mathrm{ml})$ was added and incubated for $5 \mathrm{~h}$. The medium was aspirated and replaced with $200 \mu \mathrm{l} /$ well of DMSO to dissolve the formazan salt formed. The color intensity of the formazan solution was measured at $570 \mathrm{~nm}$ by a microplate spectrophotometer. The cell viability was expressed as \% of the control (as 100\%).

Scratch motility (wound-healing) assay. HUVECs were incubated in 6-well plates and allowed to grow to full confluence. After serum starvation for $4 \mathrm{~h}$, the cells were scratched using pipette tips, washed with phosphate-buffered saline (PBS) and photographed by using a phase-contrast microscope. Fresh medium supplemented with $1 \%$ FBS and $4 \mu \mathrm{M}$ SAL was added into the well. VEGF (200 ng/ml) and $5 \mu \mathrm{M}$ cisplatin were employed as the positive and negative control, respectively. After $48 \mathrm{~h}$ of treatment, cells were photographed again at three random areas. The migrated cells were quantified by manual counting and the migration ratio was calculated.

Transwell invasion assay. HUVECs were placed on a Transwell Boyden chamber ( $8-\mu \mathrm{m}$ pore; Corning Inc., Lowell, MA, USA) pre-coated with Matrigel for $4 \mathrm{~h}$ at $37^{\circ} \mathrm{C}$. One hundred microliters of cell suspension $\left(2 \times 10^{5} \mathrm{cells} / \mathrm{ml}\right)$ in FBS-free medium was added into the upper compartment of the chamber. The bottom chambers were supplemented with $500 \mu 1$ complete medium (10\% FBS) containing the indicated concentrations of SAL with or without $200 \mathrm{ng} / \mathrm{ml}$ VEGF. After $24 \mathrm{~h}$ of treatment, the non-invaded cells from the upper face were scraped using a cotton swab. The invaded cells on the lower face were fixed with methanol, stained with Giemsa and photographed by a phase-contrast microscope. The invaded cells were quantified by manual counting and the invasion ratio was expressed as $\%$ of the control.

Tube formation assay. HUVECs $\left(1 \times 10^{4}\right.$ cells/well) were seeded in a Matrigel pre-coated 48 -well plate. VEGF $(200 \mathrm{ng} / \mathrm{ml})$ and $5 \mu \mathrm{M}$ cisplatin were employed as the positive and negative control, respectively. After $24 \mathrm{~h}$ of treatment, the tube formation was visualized with an inverted microscope and the tube number was quantified by manual counting.

Western blotting. After treatment, the cells were harvested and incubated with cell lysis buffer overnight at $-20^{\circ} \mathrm{C}$. The protein concentrations were detected using a BCA protein assay kit. After electrophoresis, the separated proteins were transferred onto nitrocellulose membrane for $75 \mathrm{~min}$ at $110 \mathrm{~V}$ and blocked with 5\% non-fat milk in TBS buffer for $1 \mathrm{~h}$. Subsequently, the membranes were washed with TBST buffer and incubated with primary antibodies [p-FAK (\#8556), VEGF (\#2463), p-VEGFR2 (\#2478s), VEGFR2 (\#9698), p-AKT (\#4058), AKT (\#4691), Ki67 (\#9027) and CD34 (\#3569); Cell Signaling Technology, Inc.] overnight at $4^{\circ} \mathrm{C}$ and then secondary antibody [IgG (\#3452), Cell Signaling Technology, Inc.] for $2 \mathrm{~h}$ at room temperature. The target proteins were detected on X-ray film using a chemiluminescence reagent. $\beta$-actin was used to confirm the equal loading and transfer of proteins.

In vivo study. Human glioma U251 cells $\left(1 \times 10^{7}\right)$ suspended in $100 \mu 1$ PBS were injected into the right lower hind flank of each 6-week-old male nude mouse. The mice were then randomly assigned into three groups of 10 mice in each group. After one week, SAL ( 5 and $10 \mathrm{mg} / \mathrm{kg}$ ) was administered into the caudal vein every other day for 16 days. Control mice received an equal volume of vehicle (saline) only. Body weight and tumor volume were monitored every two days. At the end of the experiments, tumors were excised, photographed, and weighed. Tumors from each group were used for western blotting and immunohistochemical (IHC) assay. All animal experiments were approved by the Animal Experimentation Ethics Committee of Taishan Medical University (no. 2015026).

Statistical analysis. Experiments were carried out at least in triplicate and the results are expressed as mean \pm SD. Statistical analysis was performed using SPSS version 13 (SPSS, Inc., Chicago, IL, USA). A difference between two groups was analyzed by two-tailed Student's t-test. Difference with $\mathrm{p}<0.05$ or $\mathrm{p}<0.01$ was considered statistically significant.

\section{Results}

SAL inhibits the proliferation of human HUVECs. Due to the essential role of HUVECs in angiogenesis, in the present study, HUVECs were chosen to evaluate the anti-angiogenic potential of SAL. Firstly, the inhibitory effect of SAL on the growth of HUVECs was determined by MTT assay. As shown 
A

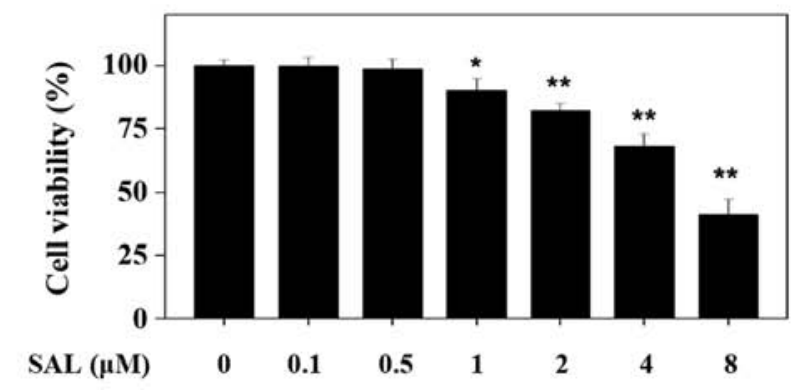

B

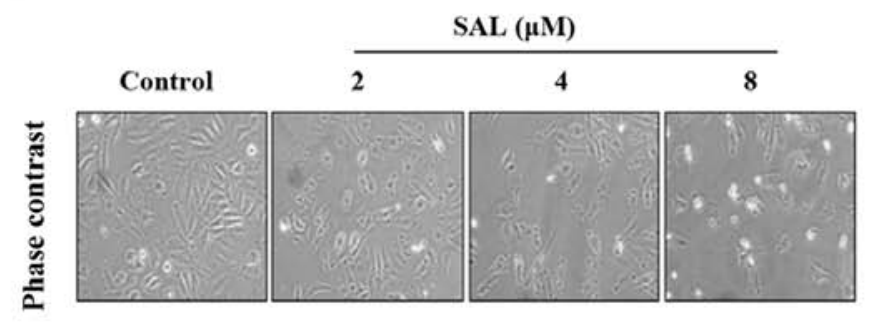

Figure 1. Salinomycin (SAL) inhibits the cell proliferation in human umbilical vein endothelial cells (HUVECs). (A) HUVECs seeded in 96-well plates (6,000 cells/well) were treated with the indicated concentrations of SAL for $48 \mathrm{~h}$, and the cell viability was detected by MTT assay. Each value represents the mean \pm SD of three independent experiments. ${ }^{*} \mathrm{p}<0.05 ;{ }^{* *} \mathrm{p}<0.01$ vs. the control. (B) Changes in the morphology of HUVECs. Cells after treatment with the indicated concentrations of SAL for $48 \mathrm{~h}$ were examined by phase-contrast microscopy (magnification, $\mathrm{x} 200$ ).

in Fig. 1A, treatment of HUVECs with the indicated concentrations $(0.1-8 \mu \mathrm{M})$ of SAL resulted in a dose-dependent inhibition of cell growth, accounting for 32.1 and $59.2 \%$ inhibition at 4 and $8 \mu \mathrm{M}$, respectively. In the phase contrast observation (Fig. 1B), HUVECs exposed to 2,4 and $8 \mu \mathrm{M}$ of SAL for $48 \mathrm{~h}$ showed a dose-dependent reduction in cell number and a change in cell morphology, such as cell shrinkage and cell rounding. These results demonstrated that SAL could inhibit HUVEC growth in vitro.

SAL blocks HUVEC migration, invasion and capillary-like tube formation. Migration, invasion and tube formation of HUVECs are crucial components in the process of tumor-induced neovascularization. Thus, we evaluated the effects of SAL on the metastatic potential of HUVECs in vitro. Scratch motility and Transwell invasive assays were used to detect the migration and invasion of HUVECs after SAL treatment, respectively. As shown in Fig. 2A-D, VEGF treatment obviously promoted HUVEC migration and invasion. However, SAL treatment effectively inhibited HUVEC migration and invasion at the concentration of $4 \mu \mathrm{M}$ after $48 \mathrm{~h}$ of treatment (Fig. 2A-D). A similar effect was also observed in the HUVECs exposed to cisplatin (negative control).

To further confirm the effect of SAL on angiogenesis, a tube formation assay was carried out to investigate whether SAL can inhibit the capillary-like tube formation of HUVECs. After pretreatment with VEGF, elongated and robust tube-like structures were observed, while a significant disruption in capillary-like tube formation was observed in the HUVECs exposed to $4 \mu \mathrm{M}$ of SAL for $24 \mathrm{~h}$ (Fig. 2E and F). These results indicated that SAL inhibited angiogenesis of HUVECs in vitro.

SAL suppresses FAK phosphorylation in HUVECs. Further investigation of the underlying mechanisms was evaluated. FAK, a cytoplasmic protein tyrosine kinase, plays a vital role in cell proliferation, survival and metastasis. To illustrate whether the inactivation of FAK is involved, we examined the levels of phosphorylated level of FAK in HUVECs after SAL treatment. As shown in Fig. 3A and B, exposure of HUVECs to SAL significantly suppressed the expression levels of phosphorylated (p)-FAK in a time- and dose-dependent manner. In addition, PF562271 (FAK inhibitor) was used to confirm the role of FAK inactivation in SAL-mediated inhibition of cell migration. As shown in Fig. 3C-E, treatment with $10 \mathrm{nM}$ PF562271 or $4 \mu \mathrm{M}$ SAL alone for $24 \mathrm{~h}$ both displayed notable inhibitory effects on FAK phosphorylation and HUVEC migration. Notably, pretreatment with PF562271 markedly enhanced SAL-induced inhibition against FAK phosphorylation and HUVEC migration. Taken together, these results indicated that FAK dephosphorylation contributed to SAK-mediated inhibition against HUVEC migration.

SAL disturbs the VEGF-VEGFR2-AKT signaling axis. As a crucial mediator of angiogenesis in the tumor microenvironment, VEGF promotes tumor angiogenesis via interacting with VEGFR to regulate downstream signaling transduction. In the present study, the expression of VEGF, VEGFR2, AKT and their phosphorylated levels in HUVECs were detected by western blotting. As shown in Fig. 4A, little change was observed in the expression levels of total VEGFR2 and AKT after SAL treatment. However, HUVECs exposed to SAL showed a significant decrease in VEGF expression, leading to the reduction of phosphorylated VERGR2 and AKT. To further study the role of AKT in SAL-mediated anti-angiogenesis in vitro, we examined the effects of AKT-upstream inhibitor (LY294002) on HUVEC growth by MTT assay. As shown in Fig. 4B, pretreatment with LY294002 resulted in a marked decrease in cell viability, which supports the key role of AKT inactivation in SAL-induced cell growth inhibition. Taken together, our results indicated that SAL inhibited HUVEC angiogenesis by disturbing the VEGF-VEGFR2-AKT signaling axis.

SAL inhibits glioma growth in vivo by inhibiting angiogenesis. To evaluate the anticancer and anti-angiogenic potential of SAL in vivo, we treated tumor-bearing nude mice with 5 and $10 \mathrm{mg} / \mathrm{kg}$ of SAL. As shown in Fig. 5A-C, the average tumor volume and tumor weight were significantly suppressed by SAL treatment. The body weight of the mice showed no significant change (Fig. 5D). Moreover, consistent with the in vitro model, we also found that SAL moderately decreased FAK and AKT phosphorylation in the tumor model (Fig. 5E). IHC staining showed that the expression of $\mathrm{Ki}-67$, a biomarker of proliferation, was significantly inhibited by SAL treatment (Fig. 5F). CD34, an important 

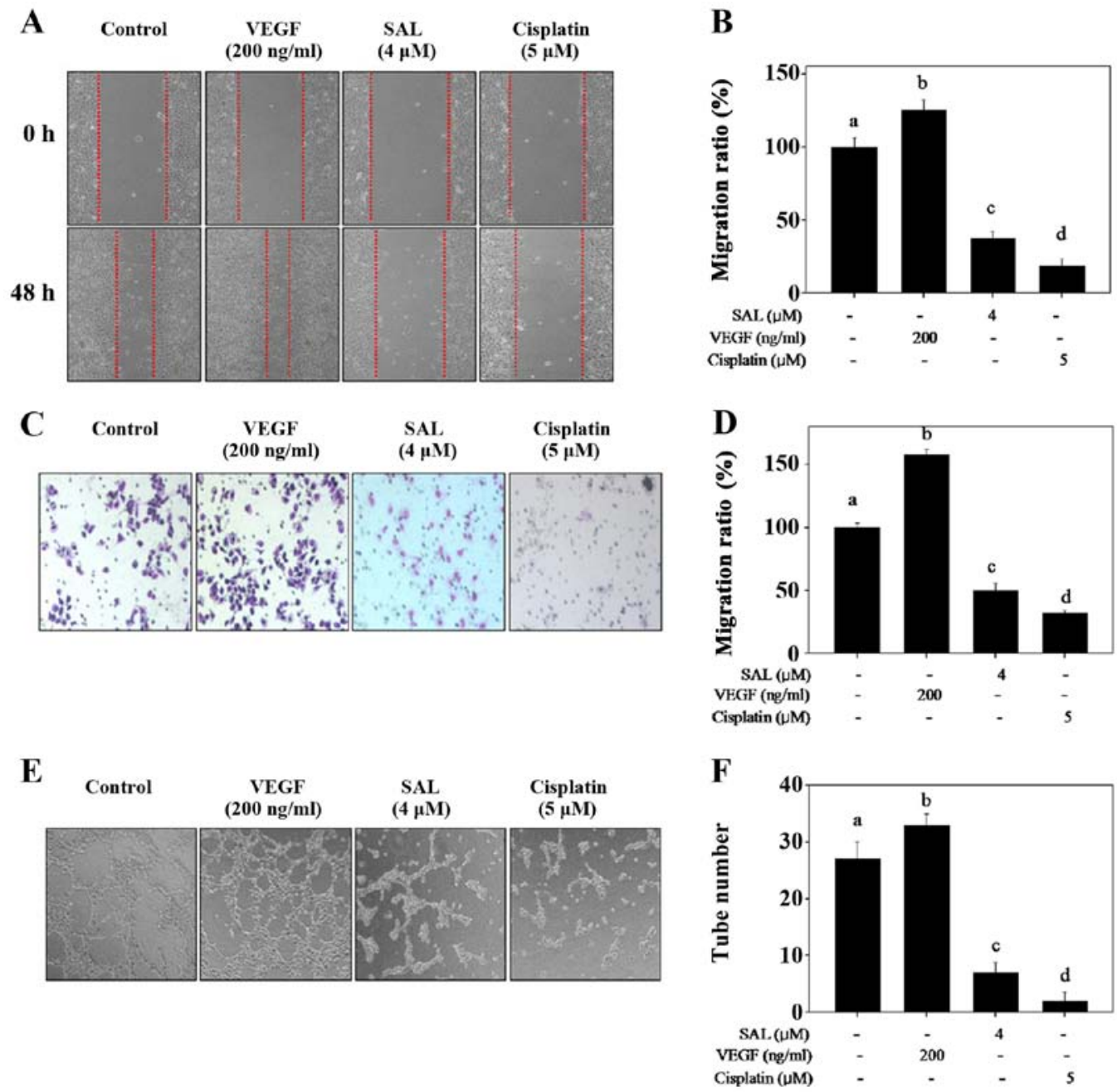

Figure 2. Salinomycin (SAL) blocks human umbilical vein endothelial cell (HUVEC) migration, invasion and capillary-like tube formation in vitro. Effects of SAL on (A) HUVEC migration, (C) invasion and (E) tube formation. Positive control [vascular endothelial growth factor (VEGF)] and negative control (cisplatin) were employed to evaluate the potential of SAL. The details of the experiments is provided in Materials and methods. (B) The migration ratio, (D) invasion rate and (F) the tube formation number were quantified by manual counting. All data are expressed as means \pm SD of triplicate experiments. Bars with different characters $(\mathrm{a}, \mathrm{b}$ and $\mathrm{c}$ ) are statistically different at $\mathrm{p}<0.05$ level.
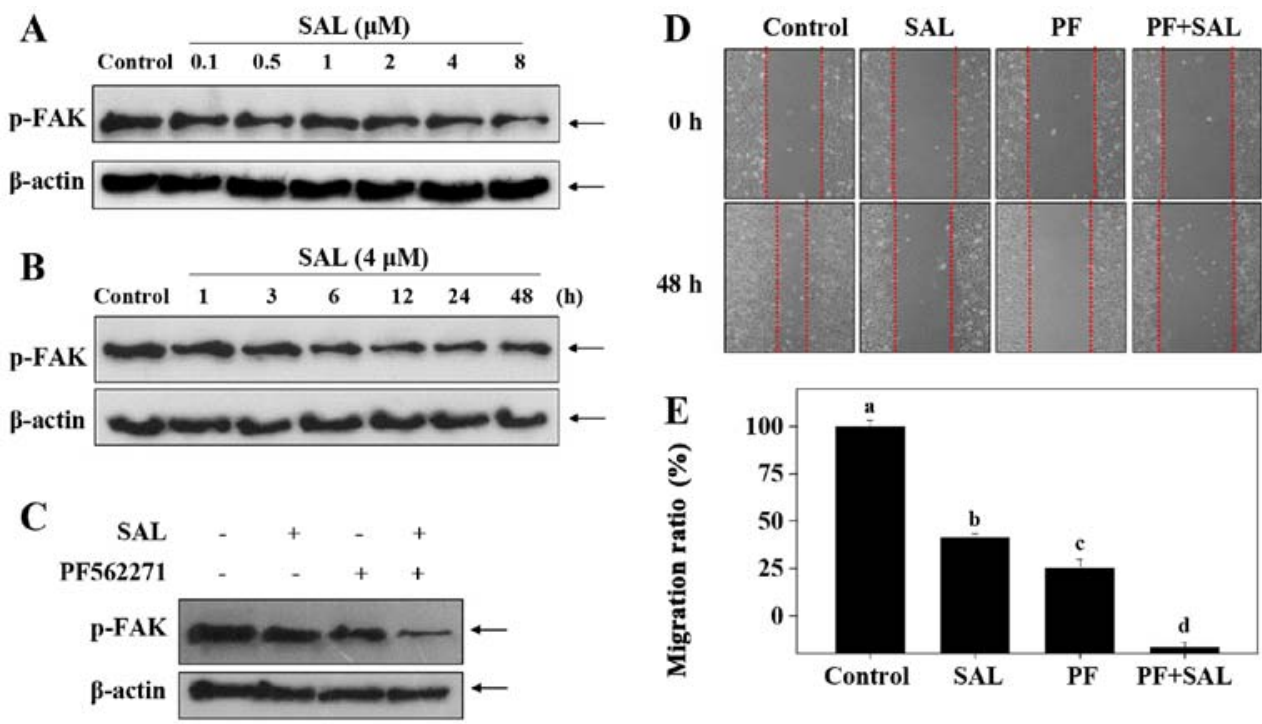

Figure 3. Salinomycin (SAL) suppresses FAK-dependent cell migration in human umbilical vein endothelial cells (HUVECs). (A) Effects of SAL on the expression level of phosphorylated FAK (p-FAK). Cells were treated with the indicated concentrations of SAL for $48 \mathrm{~h}$. (B) Western blotting of the time-dependent regulation of p-FAK in HUVECs. Cells were treated with $4 \mu \mathrm{M}$ SAL for various periods of time. (C-E) Inhibitory effects of SAL and/or FAK inhibitor (PF562271) on the expression levels of p-FAK and cell migration in HUVECs. Cells were pretreated with or without $10 \mathrm{nM}$ PF562271 for $1 \mathrm{~h}$ and co-treated with $4 \mu \mathrm{M}$ of SAL for $48 \mathrm{~h}$. All data are expressed as means \pm SD of triplicate experiments. Bars with different characters (a, b and c) are statistically different at $p<0.05$ level. 

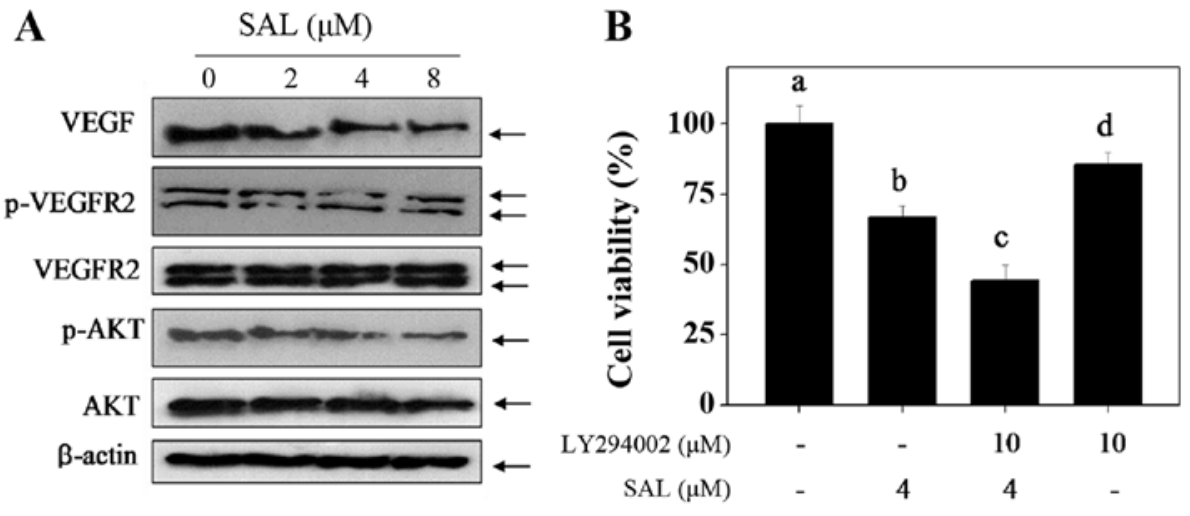

Figure 4. Salinomycin (SAL) disturbs the vascular endothelial growth factor (VEGF)-VEGFR2-AKT signaling axis. (A) Western blotting of expression levels of VEGF, VEGFR2, phosphorylated VEGFR2 (p-VEGFR2), AKT and p-AKT in human umbilical vein endothelial cells (HUVECs) exposed to different concentrations of SAL for $48 \mathrm{~h}$. (B) Inhibitory effects of SAL and/or LY294002 on HUVEC growth. Cells were pretreated with or without $10 \mu \mathrm{M} \mathrm{LY} 294002$ for $1 \mathrm{~h}$ and co-treated with $4 \mu \mathrm{M}$ of SAL for $48 \mathrm{~h}$. Cell viability was determined by MTT assay. All data are expressed as means \pm SD of triplicate experiments. Bars with different characters $(\mathrm{a}, \mathrm{b}$ and $\mathrm{c}$ ) are statistically different at $\mathrm{p}<0.05$ level.

A

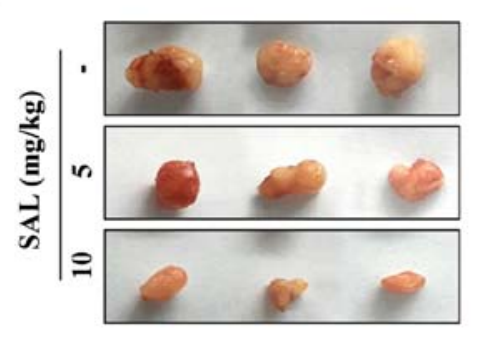

C

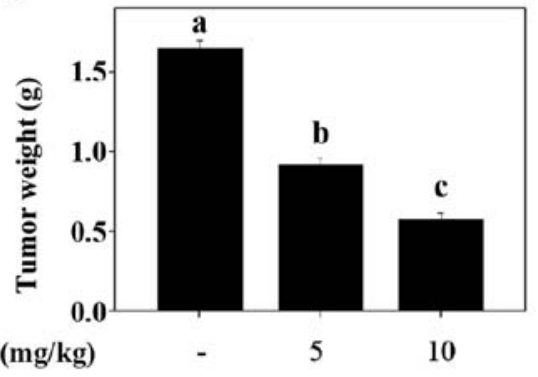

$\mathbf{E}$

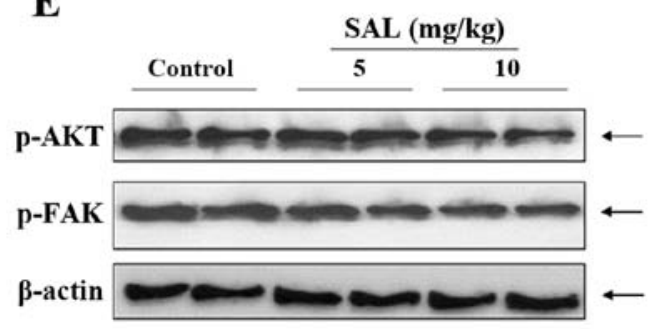

B

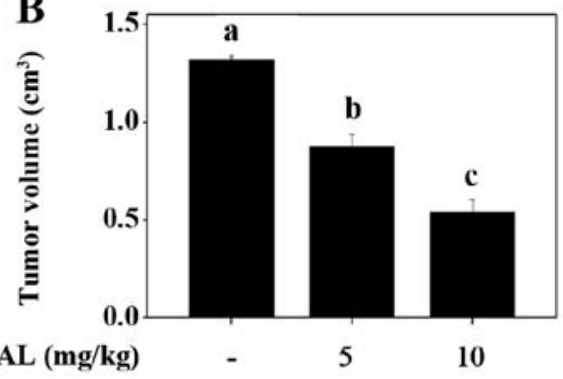

D

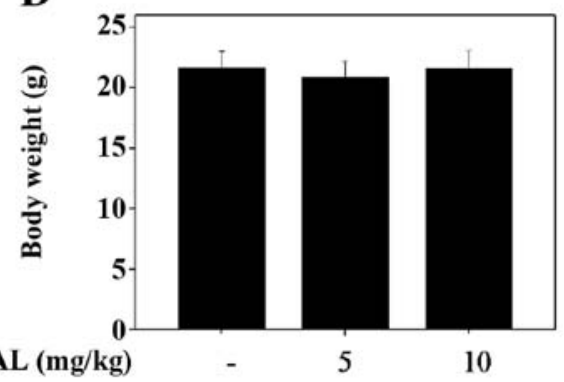

F

Ki-67

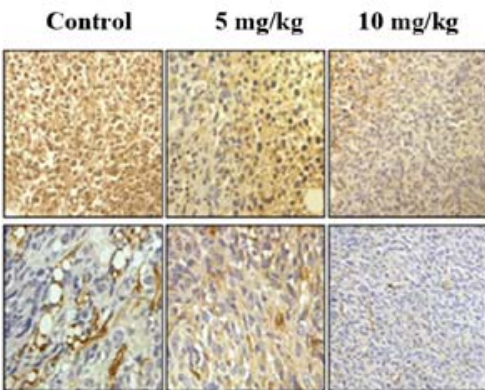

Figure 5. Salinomycin (SAL) suppresses the U251 human glioma cell growth in vivo via inhibition of angiogenesis. (A and B) Tumor volume, (C) tumor weight and (D) body weight of the mice were all assessed at the end of the experiment. U251 tumor xenograft model was established as described in Materials and methods. (E) AKT and FAK expression. Homogenized cell lysate from each group were prepared for western blotting of expression levels of phosphorylated AKT (p-AKT) and p-FAK. (F) Immunohistochemical (IHC) analysis of the expression levels of Ki-67 and CD34 in SAL-treated U251 tumor xenografts.

marker of hematopoietic progenitor cells and the small vessel endothelium was highly expressed in the control tumor tissue (Fig. 5F). However, SAL treatment significantly inhibited the in vivo angiogenesis (Fig. $5 \mathrm{~F}$ ). Taken together, 
these findings revealed that SAL hindered the U251 human glioma cell growth in vivo via inhibition of angiogenesis with involvement of AKT and FAK dephosphorylation.

\section{Discussion}

Glioma is one of the most common malignant brain cancers worldwide and has become a great threat to human health (29). Glioma is highly aggressive and is associated with a very poor prognosis, and the median survival rate is $\sim 12-15$ months $(30,31)$. Even though the current clinical treatments for glioma have achieved satisfactory results, adjuvant chemotherapy after surgical resection and radiotherapy are still irreplaceable (32-34). This forms a rationale for the development of novel chemotherapeutic agents for the treatment of glioma. Preclinical data indicate that angiogenesis is essential for the proliferation and survival of glioma cells $(35,36)$. Thus, searching for novel anti-angiogenic agents to treat human glioma is urgently needed. Herein, in the present study, we investigated the anti-angiogenic potential of SAL in the treatment of human glioma and clarify the underlying mechanisms.

Tumor cells can release growth factors and cytokines into the microenvironment, which activate the sprouting and proliferation of formerly quiescent endothelial cells on nearby blood vessels (3). VEGF, as one of the most crucial mediators in angiogenesis, can effectively promote the metastasis of endothelial cells. HUVEC migration, invasion and tube formation were significantly enhanced by the addition of VEGF. However, this tendency was significantly suppressed by SAL, indicating the anti-angiogenic potential of SAL in vitro. Growing evidence suggests that the interaction of VEGF and receptors (VEGFR1/2/3) promote the activation of downstream signaling transduction cascades $(37,38)$. Among these VEGF receptors, VEGFR2 plays a major role in the transduction of angiogenic signaling. The activation of VEGFR2 by VEGF stimulation subsequently results in activation of several downstream pathways, including Ras/MEK/ERK and PI3K/Akt, which can positively regulate pro-survival and pro-angiogenic signals $(3,37)$. Recent research has shown that SAL decreases VEGF-induced phosphorylation of VEGFR2 in HUVECs (17). Consistent with the previous data, we found that the expression levels of VEGF and phosphorylated VEGFR2 were both downregulated by the treatment of SAL, leading to the inactivation of the pro-survival PI3K/Akt signaling pathway (Fig. 4). In addition, pretreatment with the PI3K inhibitor (LY294002) markedly enhanced the growth inhibitory effects of SAL, indicating that the VEGF-VEGFR2-AKT signaling axis plays an essential role in SAL-induced HUVEC growth inhibition in vitro.

FAK, a cytoplasmic protein tyrosine kinase, plays a vital role in cell proliferation, survival and migration, and is associated with integrin-mediated signal transduction (39). Integrin clustering-mediated activation of FAK results in the phosphorylation of Tyr397, which is a binding site for PI3K (40). Previous studies have shown that the activation of FAK by integrin engagement or growth factor stimulation both promote the activation of downstream PI3K/Akt signaling pathways, which leads to the activation or overexpression of pro-metastatic proteins, such as matrix metalloproteinases, urokinase-type plasminogen activator and VEGF $(39,41)$.
Inactivation of FAK was involved in the SAL-induced suppression of metastatic potential in HUVECs (Fig 3). Moreover, the introduction of FAK inhibitor (PF562271) further confirmed the above result.

Accumulative studies have demonstrated that SAL has the ability to inhibit cancer cell growth in nude mouse xenograft models, including human gastric cancer (17), human nasopharyngeal carcinoma (18) and human hepatocellular carcinoma (19) through induction of apoptotic cell death and anti-angiogenesis. To make the in vitro data more convincible, we detected the inhibitory effect of SAL on U251 tumor xenografts. The results revealed that SAL effectively inhibited human glioma growth by inhibiting FAK and AKT phosphorylation and angiogenesis, which validated that the anti-angiogenic potential of SAL contributes to SAL-induced growth inhibition of U251 human glioma cells in vivo.

SAL is a potential anticancer agent, and its action mechanism has been extentively expolored. For example, Qin et al found that SAL inhibited human glioma cell growth by ROS/p53/cyclophilin-D signaling-mediated necrosis (42). Zhang et al found that SAL can inhibit the growth of colorectal carcinoma by targeting tumor stem cells (43). Kim et al reported that SAL can induce apoptosis and autophagy, and also acts as a sensitizer to enhance the effects of doxorubicin on human cancer cells $(20,23)$. Researchers also found that ROS-mediated oxidative damage (20,23), mitochondrial dysfunction (24), and several signaling pathway, such as FOXO3a, Wnt/ $\beta$-catenin, STAT3/ Skp2 and Akt/NF-kB/mTOR (25-28), all contribute to the anticancer mechanisms of SAL. However, we provide new evidence that SAL can act as a potent anti-angiogenic agent in vitro and in vivo to inhibit human glioma growth via suppression of the VEGF-VEGFR2-AKT/FAK signaling axis. Our findings validate the potential of SAL as a promising anti-angiogenic candidate for the treatment of human glioma in clinical trails.

\section{Acknowledgements}

This study was supported by the Science and Technology Research Projects of Shandong, China (no. 2012GSF2180 to Shi-Liang Jiang).

\section{References}

1. Sautès-Fridman C, Cherfils-Vicini J, Damotte D, Fisson S, Fridman WH, Cremer I and Dieu-Nosjean MC: Tumor microenvironment is multifaceted. Cancer Metastasis Rev 30: 13-25, 2011.

2. Valastyan S and Weinberg RA: Tumor metastasis: molecular insights and evolving paradigms. Cell 147: 275-292, 2011.

3. Weis SM and Cheresh DA: Tumor angiogenesis: molecular pathways and therapeutic targets. Nat Med 17: 1359-1370, 2011.

4. Whiteside TL: The tumor microenvironment and its role in promoting tumor growth. Oncogene 27: 5904-5912, 2008.

5. Wood SL, Pernemalm M, Crosbie PA and Whetton AD: The role of the tumor-microenvironment in lung cancer-metastasis and its relationship to potential therapeutic targets. Cancer Treat Rev 40: 558-566, 2014.

6. Fokas E, McKenna WG and Muschel RJ: The impact of tumor microenvironment on cancer treatment and its modulation by direct and indirect antivascular strategies. Cancer Metastasis Rev 31: 823-842, 2012.

7. Albini A and Sporn MB: The tumour microenvironment as a target for chemoprevention. Nat Rev Cancer 7: 139-147, 2007.

8. Fang H and Declerck YA: Targeting the tumor microenvironment: from understanding pathways to effective clinical trials. Cancer Res 73: 4965-4977, 2013. 
9. Nguyen L, Fifis T and Christophi C: Vascular disruptive agent OXi4503 and anti-angiogenic agent Sunitinib combination treatment prolong survival of mice with CRC liver metastasis. BMC Cancer 16: 533, 2016.

10. Miyazaki Y, Shibuya M, Sugawara H, Kawaguchi O and Hirsoe C: Salinomycin, a new polyether antibiotic. J Antibiot (Tokyo) 27: 814-821, 1974.

11. Gupta PB, Onder TT, Jiang G, Tao K, Kuperwasser C, Weinberg RA and Lander ES: Identification of selective inhibitors of cancer stem cells by high-throughput screening. Cell 138: 645-659, 2009.

12. Dong TT, Zhou HM, Wang LL, Feng B, Lv B and Zheng MH: Salinomycin selectively targets ' $\mathrm{CD} 133^{+'}$ cell subpopulations and decreases malignant traits in colorectal cancer lines. Ann Surg Oncol 18: 1797-1804, 2011.

13. Wang Y: Effects of salinomycin on cancer stem cell in human lung adenocarcinoma A549 cells. Med Chem 7: 106-111, 2011.

14. Zhi QM, Chen XH, Ji J, Zhang JN, Li JF, Cai Q, Liu BY, Gu QL, Zhu ZG and Yu YY: Salinomycin can effectively kill ALDH(high) stem-like cells on gastric cancer. Biomed Pharmacother 65: 509-515, 2011.

15. Zhang GN, Liang Y, Zhou LJ, Chen SP, Chen G, Zhang TP, Kang $\mathrm{T}$ and Zhao YP: Combination of salinomycin and gemcitabine eliminates pancreatic cancer cells. Cancer Lett 313 137-144, 2011.

16. Tang QL, Zhao ZQ, Li JC, Liang Y, Yin JQ, Zou CY, Xie XB, Zeng YX, Shen JN, Kang T, et al: Salinomycin inhibits osteosarcoma by targeting its tumor stem cells. Cancer Lett 311: 113-121, 2011.

17. Li T, Liu X, Shen Q, Yang W, Huo Z, Liu Q, Jiao H and Chen J: Salinomycin exerts anti-angiogenic and anti-tumorigenic activities by inhibiting vascular endothelial growth factor receptor 2-mediated angiogenesis. Oncotarget 7: 26580-26592, 2016

18. Wu D, Zhang Y, Huang J, Fan Z, Shi F and Wang S: Salinomycin inhibits proliferation and induces apoptosis of human nasopharyngeal carcinoma cell in vitro and suppresses tumor growth in vivo. Biochem Biophys Res Commun 443: 712-717, 2014.

19. Wang F, He L, Dai WQ, Xu YP, Wu D, Lin CL, Wu SM, Cheng P, Zhang Y, Shen M, et al: Salinomycin inhibits proliferation and induces apoptosis of human hepatocellular carcinoma cells in vitro and in vivo. PLoS One 7: e50638, 2012.

20. Kim SH, Choi YJ, Kim KY, Yu SN, Seo YK, Chun SS, Noh KT, Suh JT and Ahn SC: Salinomycin simultaneously induces apoptosis and autophagy through generation of reactive oxygen species in osteosarcoma U2OS cells. Biochem Biophys Res Commun 473: 607-613, 2016

21. He L, Wang F, Dai WQ, Wu D, Lin CL, Wu SM, Cheng P, Zhang Y, Shen M, Wang CF, et al: Mechanism of action of salinomycin on growth and migration in pancreatic cancer cell lines. Pancreatology 13: 72-78, 2013.

22. Kim KY, Kim SH, Yu SN, Park SK, Choi HD, Yu HS, Ji JH Seo YK and Ahn SC: Salinomycin enhances doxorubicin-induced cytotoxicity in multidrug resistant MCF-7/MDR human breast cancer cells via decreased efflux of doxorubicin. Mol Med Rep 12: 1898-1904, 2015.

23. Kim JH, Chae M, Kim WK, Kim YJ, Kang HS, Kim HS and Yoon S: Salinomycin sensitizes cancer cells to the effects of doxorubicin and etoposide treatment by increasing DNA damage and reducing p21 protein. Br J Pharmacol 162: 773-784, 2011.

24. Managò A, Leanza L, Carraretto L, Sassi N, Grancara S, Quintana-Cabrera R, Trimarco V, Toninello A, Scorrano L, Trentin L, et al: Early effects of the antineoplastic agent salinomycin on mitochondrial function. Cell Death Dis 6 : e1930, 2015 .
25. Lu D, Choi MY, Yu J, Castro JE, Kipps TJ and Carson DA Salinomycin inhibits Wnt signaling and selectively induces apoptosis in chronic lymphocytic leukemia cells. Proc Natl Acad Sci USA 108: 13253-13257, 2011.

26. Zhou Y, Liang C, Xue F, Chen W, Zhi X, Feng X, Bai X and Liang T: Salinomycin decreases doxorubicin resistance in hepatocellular carcinoma cells by inhibiting the $\beta$-catenin/TCF complex association via FOXO3a activation. Oncotarget 6: 10350-10365, 2015.

27. Koo KH, Kim H, Bae YK, Kim K, Park BK, Lee CH and Kim YN: Salinomycin induces cell death via inactivation of Stat3 and downregulation of Skp2. Cell Death Dis 4: e693, 2013.

28. Parajuli B, Lee HG, Kwon SH, Cha SD, Shin SJ, Lee GH, Bae I and $\mathrm{Cho} \mathrm{CH}$ : Salinomycin inhibits $\mathrm{Akt} / \mathrm{NF}-\kappa \mathrm{B}$ and induces apoptosis in cisplatin resistant ovarian cancer cells. Cancer Epidemiol 37: 512-517, 2013.

29. Huse JT and Holland EC: Targeting brain cancer: advances in the molecular pathology of malignant glioma and medulloblastoma. Nat Rev Cancer 10: 319-331, 2010

30. Ho AL, Koch MJ, Tanaka S, Eichler AF, Batchelor TT, Tanboon J, Louis DN, Cahill DP, Chi AS and Curry WT Jr: Impact of histopathological transformation and overall survival in patients with progressive anaplastic glioma. J Clin Neurosci 31: 99-105, 2016

31. Dewan MC, White-Dzuro GA, Brinson PR, Thompson RC and Chambless LB: Perioperative seizure in patients with glioma is associated with longer hospitalization, higher readmission, and decreased overall survival. J Neurosurg 125: 1033-1041, 2016.

32. Schaff LR and Lassman AB: Indications for treatment: is observation or chemotherapy alone a reasonable approach in the management of low-grade gliomas? Semin Radiat Oncol 25: 203-209, 2015.

33. Blondin NA and Becker KP: Anaplastic gliomas: radiation, chemotherapy, or both? Hematol Oncol Clin North Am 26: 811-823, 2012.

34. Viaccoz A, Lekoubou A and Ducray F: Chemotherapy in low-grade gliomas. Curr Opin Oncol 24: 694-701, 2012.

35. Onishi M, Ichikawa T, Kurozumi K and Date I: Angiogenesis and invasion in glioma. Brain Tumor Pathol 28: 13-24, 2011.

36. Tate MC and Aghi MK: Biology of angiogenesis and invasion in glioma. Neurotherapeutics 6: 447-457, 2009.

37. Kerbel RS: Tumor angiogenesis. N Engl J Med 358: 2039-2049, 2008

38. Liang X, Xu F, Li X, Ma C, Zhang Y and Xu W: VEGF signal system: the application of antiangiogenesis. Curr Med Chem 21: 894-910, 2014.

39. Zhang J and Hochwald SN: The role of FAK in tumor metabolism and therapy. Pharmacol Ther 142: 154-163, 2014.

40. Chen HC, Appeddu PA, Isoda H and Guan JL: Phosphorylation of tyrosine 397 in focal adhesion kinase is required for binding phosphatidylinositol 3-kinase. J Biol Chem 271: 26329-26334, 1996.

41. Smith HW and Marshall CJ: Regulation of cell signalling by uPAR. Nat Rev Mol Cell Biol 11: 23-36, 2010.

42. Qin LS, Jia PF, Zhang ZQ and Zhang SM: ROS-p53-cyclophilin-D signaling mediates salinomycin-induced glioma cell necrosis. J Exp Clin Cancer Res 34: 57, 2015.

43. Zhang C, Tian Y, Song F, Fu C, Han B and Wang Y: Salinomycin inhibits the growth of colorectal carcinoma by targeting tumor stem cells. Oncol Rep 34: 2469-2476, 2015. 